\title{
The test-retest reliability of three different hip joint centre location techniques
}

\author{
J Sinclair $^{1}$, PJ Taylor ${ }^{2}$, G Currigan ${ }^{1}$ and SJ Hobbs ${ }^{1}$ \\ 1 Division of Sport Exercise and Nutritional Sciences, University of Central Lancashire \\ 2 School of Psychology, University of Central Lancashire
}

Received February 24th 2013 - Accepted June 3rd 2013

\begin{abstract}
The hip joint centre (HJC) is essential in kinematic analyses. Three principal techniques exist for the estimation of the HJC, anatomical, functional and projection, although their test-retest has not been established. Fifteen participants ran at $4.0 \mathrm{~m} . \mathrm{s}^{-1} \pm 5 \%$; 3 -D kinematics of the hip and knee were collected. The HJC was defined twice using each technique (test-and-retest) and the kinematic data was applied to both. The results show significant test-retest differences in the coronal and transverse planes using the functional and projection techniques. Strong test-retest intraclasscorrelations were observed for all techniques although the anatomical method was associated with the highest reliability. The anatomical technique for HJC estimation appears to be the most reliable; therefore its utilization for 3-D analyses is recommended.
\end{abstract}

Key words: Hip joint centre, 3-D kinematics, reliability

Résumé. Tests de reproductibilité pour trois méthodes de positionnement du centre articulaire de la hanche.

Le positionnement du centre articulaire de la hanche (HJC) est essentiel dans les analyses cinématiques. Trois techniques principales existent pour l'évaluation du HJC : anatomique, fonctionnelle et de projection mais la reproductibilité de ces méthodes n'a jamais été vérifiée. Quinze participants ont couru à une vitesse égale à $4,0 \pm 5 \% \mathrm{~m} \cdot \mathrm{s}^{-1}$. L'analyse cinématique 3 -D au niveau de la hanche et le genou a été réalisée et le HJC a été déterminé deux fois pour chaque technique (test et re-test). Les résultats montrent des différences significatives de test-retest dans les plans frontaux et transversaux pour les techniques fonctionnelles et de projection. De bonnes test-retest intraclass corrélations ont été observées pour les trois techniques mais la méthode anatomique présentait la plus grande reproductibilité. La technique anatomique pour l'estimation du HJC semble donc la plus pertinente pour une analyse cinématique $3-\mathrm{D}$.

Mots clés : Centre articulaire de la hanche, cinématique à trois dimensions, reproductibilité

\section{Introduction}

The hip joint centre (HJC) landmark is critical in human movement analyses as it allows the determination of the anatomical reference frame of the femur. Accurately locating the HJC is required to accurately quantify hip and knee joint rotations in gait analyses (Capozzo, Leo, \& Pedotti, 1975; Kirkwood, Culham, \& Costigan, 1999; Stagni, et al., 1987).

A number of techniques currently exist for the estimation of the HJC which includes the anatomical, method whereby the position of the HJC is estimated relative to anatomic pelvic landmarks (Andriacchi, Anderson, Fermier, Stern, \& Galante, 1980; Bell, Brand, \& Pedersen,
1999; Seidel, Marchinda, Dijkers, \& Soutas-Little, 1995; Tylkowski., Simon, \& Mansour, 1982) Researchers have also developed the functional technique that estimates the centre of rotation of the thigh segment relative to the pelvis using 3-D kinematic data (Cappozzo, 1984; Leardini, et al., 1999; Marin, Mannel, Claes, \& Durselen, 2003; Piazza, Okita, \& Cavanagh, 2001; Shea, Lenhoff, Otis, \& Backus, 1997). Finally a projection technique method places the HJC at one-quarter of the distance from the ipsolateral to the contralateral greater trochanter has also been developed and advocated by researchers (Weinhandl \& O'Connor, 2010).

Although the validity of each technique has been reported to justify their utilization, there is currently a lack 
of consensus regarding the most appropriate technique for running analyses (Besier, Sturnieks, Alderson, \& Lloyd, 2003). Furthermore, whilst investigations have been conducted to examine the reliability of the anatomical position of the HJC, there is a paucity of information regarding the influence that the different techniques have upon the reliability of the resultant hip and knee joint kinematic waveforms and discrete 3-D kinematic variables. Therefore the aim of the current investigation is to compare the test-retest reliability in 3-D hip and knee joint kinematics using the three (anatomical, projection and functional) different HJC estimation techniques currently utilized within the literature using both discrete variable and waveform analyses.

\section{Methods}

\subsection{Participants}

Fifteen male participants volunteered to take part in this investigation. All were free from musculoskeletal pathology at the time of data collection and provided written informed consent in accordance with the declaration of Helsinki. The mean characteristics of the participants were; age $24.2 \pm 5.4$ years, height $1.77 \pm 0.07 \mathrm{~m}$, body mass $70.4 \pm 6.6 \mathrm{~kg}$, BMI $22.3 \pm 1.4$ and body fat $\%$ $10.5 \pm 3.2$. An a priori statistical power analysis was conducted using the Hopkins method based on a moderate effect size and a power measure of $80 \%$, which suggested that 15 participants were adequate for the design. Ethical approval for this project was obtained from the University of Central Lancashire School of Sport Tourism and Outdoors ethical committee.

\subsection{Procedure}

Participants ran at $4.0 \mathrm{~m} . \mathrm{s}^{-1}$, striking a force plate (Kistler, Kistler Instruments Ltd., Alton, Hampshire) embedded in the floor (Altrosports 6mm, Altro Ltd,) of a $22 \mathrm{~m}$ biomechanics laboratory with their dominant (Right) limb. Running velocity was quantified using Newtest 300 infrared timing gates (Newtest, Oy Koulukatu, Finland); a maximum deviation of $\pm 5 \%$ from the set velocity was allowed. Stance time was defined as the time over which $20 \mathrm{~N}$ or greater of vertical force was applied to the force platform (Sinclair, Edmundson, Brooks, \& Hobbs, 2011). A successful trial was defined as one within the specified velocity range, where all tracking clusters were in view of the cameras, the foot made full contact with the force plate and no evidence of gait modification due to the experimental conditions.

All kinematic data were captured at $250 \mathrm{~Hz}$ using an eight camera motion analysis system (Qualisys ${ }^{\mathrm{TM}}$ Medical AB, Goteburg, Sweden). The marker configuration for this investigation used the calibrated anatomical systems technique (CAST) technique (Cappozzo, Catani,
Leardini, Benedeti, \& Della, 1995). Retro-reflective markers $(19 \mathrm{~mm})$ were positioned on the participant's lower limbs. Technical tracking clusters comprising of four $19 \mathrm{~mm}$ spherical retro-reflective markers mounted to a thin sheath of lightweight carbon fiber, were positioned on the shank, thigh and pelvis. The tracking clusters had length to width ratios of 2.05:1-1.5:1, in accordance previous recommendations (Cappozzo, Cappello, Croce, \& Pensalfini, 1997). In order to provide anatomical meaning for the technical markers and define the pelvis, right thigh and right shank segments retro-reflective markers were positioned on the medial and lateral malleoli, medial and lateral epicondyle of the femur, greater trochanter of the right leg, anterior superior iliac spines (ASIS) and posterior superior iliac spines (PSIS). For all segments, the positive $\mathrm{Z}$ (transverse plane) axis was defined in the direction of distal to proximal joint centres. The positive Y (coronal plane) axis was defined as perpendicular to the $\mathrm{Z}$ axis and while the $\mathrm{X}$ (sagittal) axis was delineated as a cross-product of $\mathrm{Y}$ and $\mathrm{Z}$ axes. All markers were positioned by the lead author.

To examine the test-retest reliability of HJC estimation via the projection and anatomical techniques two static calibration trials were captured with the participant standing in the anatomical position allowing the positions of the anatomical markers to be referenced in relation to the tracking clusters. The anatomical technique was based on previously outlined recommendations (Bell, Brand, \& Pedersen, 1999) via the inter-ASIS breadth. This method placed the HJC $14 \%$ of the ASIS breadth medially, $19 \%$ posteriorly, and $30 \%$ distally from the ipsilateral (Right) ASIS. The projection technique was also based on previously established recommendations (Weinhandl, \& O'Connor, 2010), this method estimates the HJC as a three-dimensional point, located at one-quarter of the distance along a line from the ipsolateral (Right) to the contralateral (Left) greater trochanter markers during the static trials. The first static (test) was conducted prior to the running trials, following which the left and right ASIS markers and right greater trochanter markers were removed. The medial and lateral femoral epicondyle and malleoli markers remained in position throughout, and were attached with strong adhesive tape to ensure that the distal end of the thigh and the proximal/distal ends of the tibia remained consistent. This allowed the reliability of the HJC to be examined. Following completion of the running trials the ASIS and greater trochanter markers were re-positioned and the second static trial (retest) was obtained.

The functional method used functional techniques based on Schwartz and Rozumalski (2005). The calculation used rotations between two the pelvis and thigh segments to calculate an instantaneous axis of rotation. The average intersection point of these axes of rotations gives an approximate centre of rotation, which then defines the joint centre. To define the functional HJC participants performed five sequential flexion-extension and abduction-adduction movements of the right hip at a 


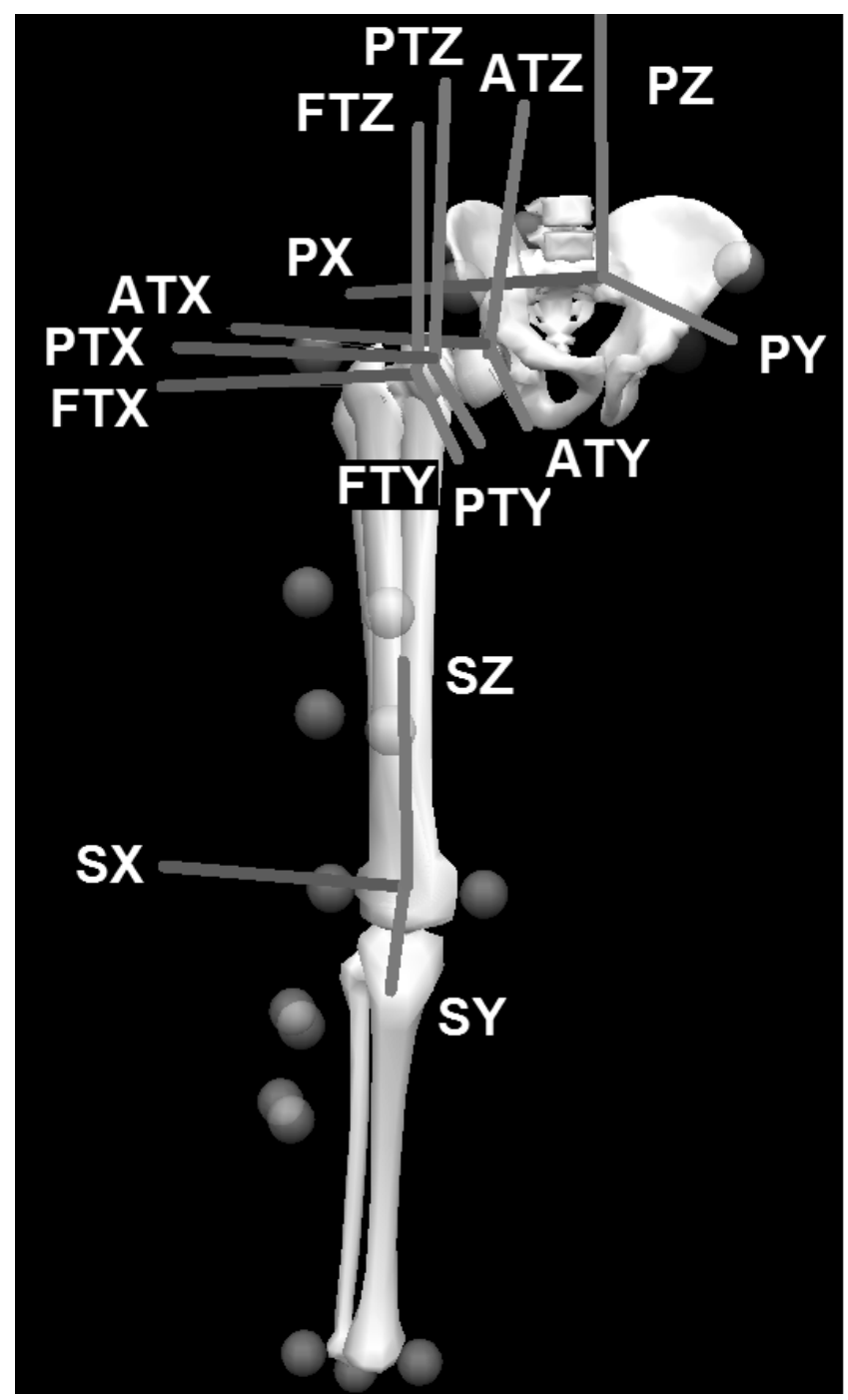

Fig. 1. Pelvic, thigh, tibial and foot segments, with segment co-ordinate system axes. $(\mathrm{P}=$ pelvis, $\mathrm{S}=$ shank, $\mathrm{AT}=$ anatomical thigh, $\mathrm{PT}=$ projection thigh and $\mathrm{FT}=$ functional thigh).

self-selected velocity followed by a cycle of full hip circumduction. Flexion-extension and abduction-adduction ranges of movement were in the order of 45 and $40^{\circ}$, respectively (Leardini, et al., 1999). To examine the repeatability of this technique, this procedure was repeated before (test) and after the collection of the running (retest) data (prior to the collection of the static trials).

Cluster markers used to define the technical tracking frame of the thigh, tibial and pelvic segments remained rigidly in place for the duration of the analysis and were not removed, allowing the test-retest reliability of the HJC location to be examined. To assess the test-retest differences in HJC location on dynamic 3-D kinematics, the same dynamic information were applied to the test and retest static trials.

\subsection{Data processing}

Trials from each HJC condition were processed using Qualisys Track Manager to identify anatomical and cluster markers. Files were then exported in C3D format. 3 -D kinematic parameters were quantified using Visual 3-D (C-Motion, Germantown, MD, USA) after the data had been smoothed via Butterworth low pass 4th order zero-lag filter at a cut off frequency of $10 \mathrm{~Hz}$ which was selected as being the frequency at which $95 \%$ of the signal power was below. Stance phase data was time normalised to 101 points. Hip, knee and ankle joint kinematics were quantified using an $X Y Z$ sequence of rotations. Kinematic waveforms were time normalized to $100 \%$ of the stance phase. 3-D kinematic measures from the hip, knee and ankle which were extracted for statistical analysis were 1) angle at footstrike, 2) angle at toe-off, 3) range of motion (ROM) from footstrike to toe-off during stance, 4) peak angle during stance, 5) relative ROM from footstrike to peak angle.

\subsection{Statistical analysis}

Descriptive statistics including means and standard deviations were calculated between test and retest parameters for each of the three HJC techniques. Differences in stance phase kinematic parameters were examined using Wilcoxon rank tests with significance accepted at the $p \leqslant 0.05$ level (Rothman, 1990) Intra-class correlations were also utilized to compare test and retest sagittal, coronal and transverse plane waveforms of the hip and knee for each HJC location technique. The average, upper and lower bound measures of these correlations were reported. Finally, cronbach's alpha $(\alpha)$ were used to examine the reliability of the discrete variables. All statistical procedures were conducted using SPSS 19.0 (SPSS Inc, Chicago, USA).

\section{Results}

The overall patterns of the test-retest 3-D kinematic waveforms were qualitatively similar for all three techniques (Figs. 1-3), although statistical differences were observed at the hip and knee for the functional and projection techniques (Tabs. 1 and 2).

The comparative results for the hip joint indicate that for the functional technique significant differences were observed in the coronal plane between test and retest for the magnitude of rotation at footstrike $Z=2.01, p \leqslant 0.05$ and peak coronal plane abduction $Z=2.10, p \leqslant 0.05$. In addition, for the projection technique significant differences were also observed in both the coronal plane for the magnitude of rotation at footstrike $Z=2.35, p \leqslant 0.05$, and peak coronal plane abduction $Z=2.20, p \leqslant 0.05$. In the transverse plane significant differences were also 

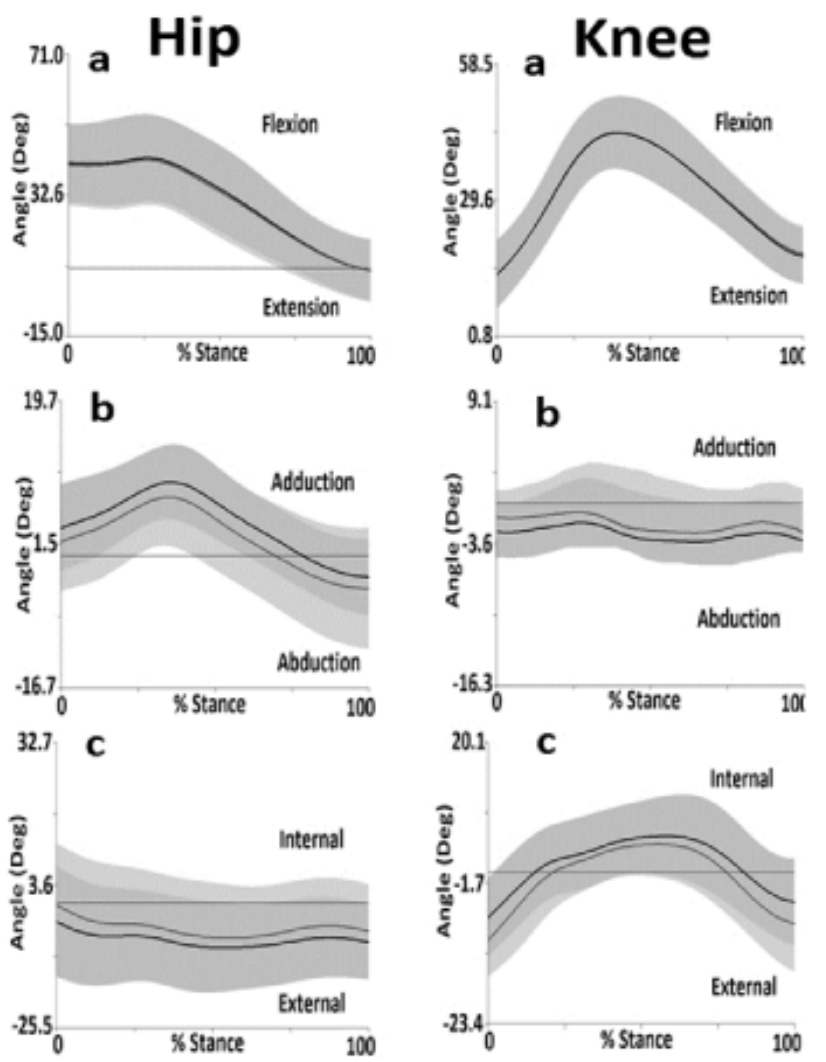

Fig. 2. Hip and knee joint kinematics from the anatomical HJC in the a. sagittal, b. coronal and c. transverse plane as a function of test (black) and retest (grey) static trials.

found when using the projection technique for the magnitudes of rotation at toe-off $Z=2.35, p \leqslant 0.05$ and peak external rotation $Z=2.36, p \leqslant 0.05$. No significant differences were observed for the anatomical technique.

Comparisons between test and retest hip joint kinematic waveforms for the anatomical technique revealed strong correlations for the sagittal $R=0.984$ (0.941-1.00), coronal $R=0.994(0.979-1.00)$ and transverse $R=0.90(0.861-0.950)$ planes. For the functional method strong correlations were observed between test and retest waveforms in the sagittal $R=0.982$ (0.905-1.00), coronal $R=0.986(0.91-1.00)$ and transverse $R=0.899(0.864-0.944)$ planes. Finally, for the projection technique strong correlations were also observed for the sagittal $R=0.974(0.851-0.995)$, coronal $R=0.968(0.845-0.995)$ and transverse $R=0.894$ planes (0.721-0.963).

The comparative results for the knee joint indicate that for the functional technique significant differences were observed in the coronal plane between test and retest for the magnitude of rotation at footstrike $Z=2.40$, $p \leqslant 0.01$, toe-off $Z, 2.50, p \leqslant 0.05$ and peak adduction $Z=2.30, p \leqslant 0.05$. In the transverse plane significant
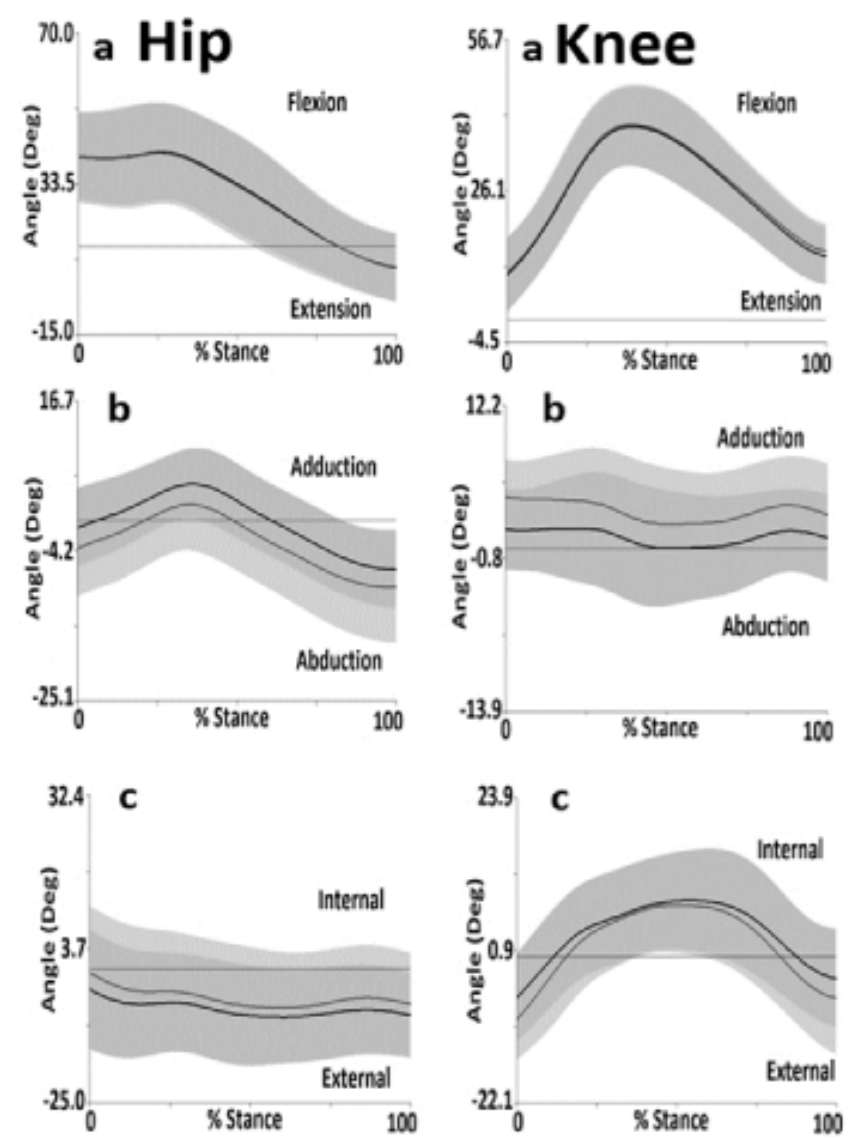

Fig. 3. Hip and knee joint kinematics from the functional HJC in the a. sagittal, b. coronal and c. transverse plane as a function of test (black) and retest (grey) static trials.

differences were observed for the magnitude of relative ROM $Z=2.23, p \leqslant 0.05$. In addition, for the projection technique significant differences were also observed in the coronal plane for the magnitudes of rotation at footstrike $Z=2.86, p \leqslant 0.01$, toe-off $Z=2.90, p \leqslant 0.01$ and peak adduction $Z=2.86, p \leqslant 0.01$. In the transverse plane significant differences were observed for the magnitude of relative $\mathrm{ROM} Z=2.79, p \leqslant 0.01$ No significant differences were observed for the anatomical technique.

Comparisons between test and retest knee joint kinematic waveforms for the anatomical technique revealed strong correlations for the sagittal $R=0.999$ (0.98-1.00), coronal $R=0.942(0.920-0.995)$ and transverse $R=0.971 \quad(0.894-0.999)$ planes. For the functional method strong correlations were observed between test and retest waveforms in the sagittal $R=0.997$ (0.860-1.00), coronal $R=0.892(0.815-0.952)$ and transverse $R=0.96(0.870-0.991)$ planes. Finally, for the projection technique strong correlations were also observed for the sagittal $R=0.990(0.86-1.00)$, coronal $R=0.838$ $(0.772-0.904)$ and transverse $R=0.861(0.801-0.932)$ planes. 
The test-retest reliability of three different hip joint centre location techniques

\begin{tabular}{|c|c|c|c|c|c|c|c|c|c|c|c|c|c|c|c|}
\hline$\gamma$ & & $\begin{array}{l}\infty \\
\infty \\
\infty \\
0 \\
0\end{array}$ & $\begin{array}{l}\stackrel{g}{\circ} \\
\stackrel{\circ}{\circ}\end{array}$ & $\begin{array}{l}\infty \\
\infty \\
\infty \\
0 \\
0\end{array}$ & 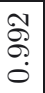 & $\begin{array}{l}0 \\
\infty \\
\infty \\
0 \\
0\end{array}$ & $\begin{array}{l}+ \\
\sigma^{\prime} \\
\infty \\
0\end{array}$ & \begin{tabular}{|l|}
$\infty$ \\
$\infty$ \\
$\infty$ \\
0 \\
0
\end{tabular} & \begin{tabular}{|l|}
$\mathfrak{N}$ \\
0 \\
0 \\
0 \\
\end{tabular} & 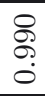 & $\begin{array}{l}\mathcal{N} \\
\infty \\
\infty \\
0 \\
0\end{array}$ & $\begin{array}{l}\infty \\
\stackrel{\infty}{o} \\
\stackrel{\sigma}{\circ}\end{array}$ & $\begin{array}{l}\stackrel{P}{1} \\
\infty \\
0 \\
0\end{array}$ & \begin{tabular}{l}
$\vec{\infty}$ \\
\multirow{0}{0}{} \\
0
\end{tabular} & $\begin{array}{l}10 \\
10 \\
\infty \\
0 \\
0\end{array}$ \\
\hline & 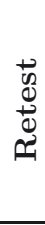 & 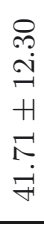 & $\begin{array}{l}\stackrel{\rho}{\infty} \\
\infty \\
+ \\
+ \\
\stackrel{1}{0} \\
\dot{0} \\
1\end{array}$ & 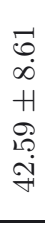 & 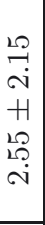 & 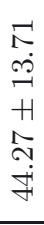 & $\begin{array}{l}\vec{\infty} \\
10 \\
+ \\
+1 \\
10 \\
-1\end{array}$ & 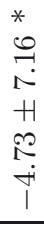 & $\begin{array}{l}0 \\
r \\
20 \\
H \\
0 \\
0 \\
0\end{array} \mid$ & 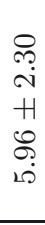 & 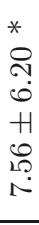 & 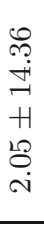 & 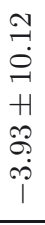 & $\begin{array}{l}0 \\
\circ \\
10 \\
+1 \\
+ \\
\infty \\
0\end{array}$ & $\begin{array}{l}\hat{\sigma} \\
\infty \\
\infty \\
H \\
\infty \\
\curvearrowright \\
\infty\end{array}$ \\
\hline & $\vec{\theta}$ & 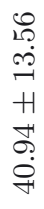 & 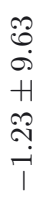 & 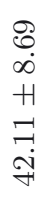 & 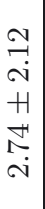 & 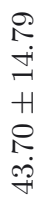 & 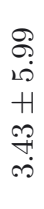 & 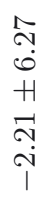 & $\mid \begin{array}{l}0 \\
0 \\
\infty \\
H \\
\infty \\
-1 \\
\underset{0}{0}\end{array}$ & $\begin{array}{l}20 \\
\sim \\
\sim \\
H \\
H \\
0 \\
\sim \\
0\end{array}$ & $\begin{array}{l}0 \\
\varrho \\
0 \\
+1 \\
\infty \\
0 \\
0\end{array}$ & 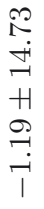 & 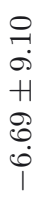 & $\begin{array}{l}10 \\
\infty 0 \\
10 \\
1 \\
10 \\
10 \\
0\end{array}$ & $\begin{array}{l}\overrightarrow{1} \\
10 \\
10 \\
H \\
10 \\
\sim \\
\infty\end{array}$ \\
\hline & & $\begin{array}{ll}10 \\
\infty\end{array}$ & $\stackrel{\infty}{\llcorner}$ & ஓ & $\stackrel{N}{\infty}$ & $\infty$ & $\infty$ & $\infty$ & \begin{tabular}{|l|}
2 \\
10
\end{tabular} & $\stackrel{12}{\mathscr{2}}$ & $\begin{array}{l}10 \\
\infty\end{array}$ & $\stackrel{\sim}{\sim}$ & $\stackrel{\sim}{\sim}$ & $\stackrel{\infty}{1}$ & $\begin{array}{l}10 \\
\infty\end{array}$ \\
\hline
\end{tabular}

\begin{tabular}{|c|c|c|c|c|c|c|c|c|c|c|c|c|c|c|}
\hline \multirow[t]{2}{*}{$\gamma$} & $\begin{array}{l}10 \\
0 \\
0 \\
0 \\
0\end{array}$ & $\begin{array}{l}\infty \\
\stackrel{\infty}{1} \\
0 \\
0 \\
0\end{array}$ & @ & $\begin{array}{l}1 \\
\infty \\
0 \\
0 \\
0\end{array}$ & $\begin{array}{l}\infty \\
\infty \\
0 \\
0\end{array}$ & $\begin{array}{l}N \\
\infty \\
\infty \\
0 \\
0\end{array}$ & $\begin{array}{l}\infty \\
0 \\
\infty \\
0 \\
0\end{array}$ & $\begin{array}{l}10 \\
20 \\
0 \\
0\end{array}$ & $\begin{array}{l}20 \\
\stackrel{2}{\circ} \\
0 \\
0\end{array}$ & \begin{tabular}{|l|}
12 \\
$\infty$ \\
$\infty$ \\
0 \\
0
\end{tabular} & 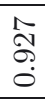 & $\begin{array}{l}\vec{N} \\
\infty \\
0 \\
0\end{array}$ & \begin{tabular}{|l} 
\\
1 \\
0 \\
0 \\
0
\end{tabular} & $\begin{array}{l}10 \\
\infty \\
0 \\
0 \\
0\end{array}$ \\
\hline & $\Rightarrow$ & 70 & & & 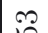 & 0 & 0 & & & $*$ & 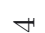 & $\because$ & & \\
\hline
\end{tabular}

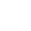

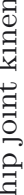

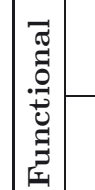

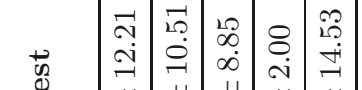

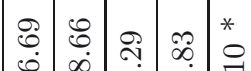

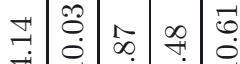

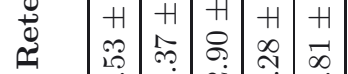

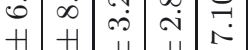

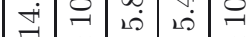

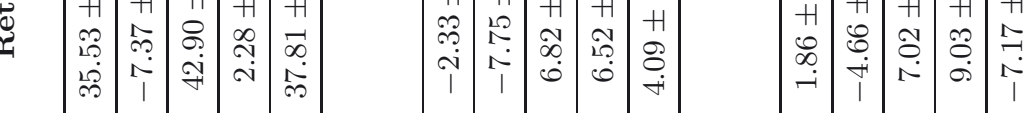

争 궁

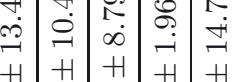

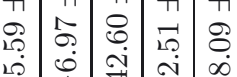

萧

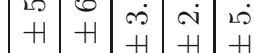

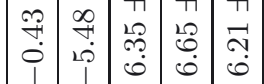

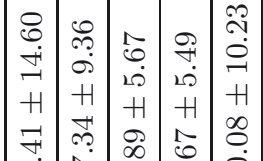

\begin{tabular}{|c|c|c|c|c|c|c|c|c|c|c|c|c|c|c|c|c|}
\hline & & $\left|\begin{array}{l}\infty \\
0 \\
\vdots \\
\vdots \\
\vdots\end{array}\right|$ & \begin{tabular}{l|l}
$\overrightarrow{0}$ & 0 \\
0 \\
$\stackrel{0}{0}$
\end{tabular} & 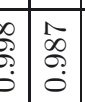 & 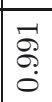 & & $\mid \begin{array}{l}\vec{\partial} \\
0 \\
\dot{0}\end{array}$ & & \begin{tabular}{|l|} 
\\
$\vdots$ \\
$\vdots$ \\
0
\end{tabular} & & $\begin{array}{l}\infty \\
\infty \\
\infty \\
\infty \\
\infty\end{array}$ & & \begin{tabular}{|c|}
\multirow{2}{*}{} \\
$\dot{a}$ \\
$\dot{0}$
\end{tabular} & 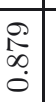 & 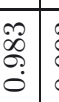 & 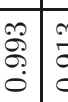 \\
\hline & 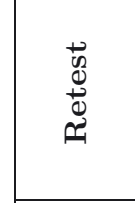 & 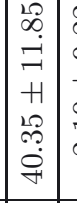 & 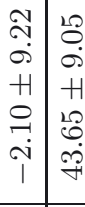 & 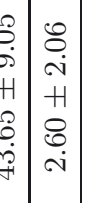 & 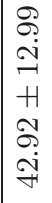 & & 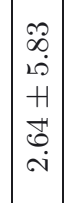 & 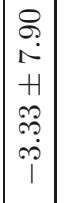 & \begin{tabular}{|c|}
0 \\
$\infty$ \\
0 \\
0 \\
$H$ \\
\\
0 \\
0 \\
$\dot{0}$
\end{tabular} & $\begin{array}{l}\overrightarrow{1} \\
\stackrel{1}{\mathrm{i}} \\
\mathrm{H} \\
\overrightarrow{\mathrm{Z}} \\
\overrightarrow{0}\end{array}$ & 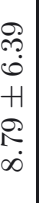 & & 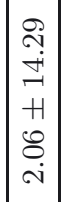 & 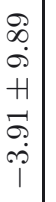 & 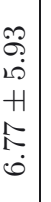 & 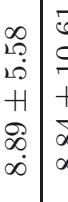 \\
\hline & $\begin{array}{l}\text { du } \\
\text { du }\end{array}$ & 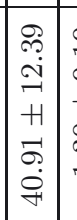 & 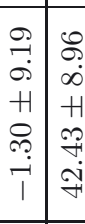 & 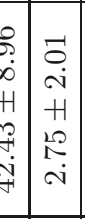 & 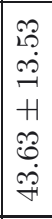 & & 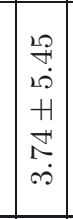 & \begin{tabular}{|c|c|}
$\infty$ \\
$\infty$ \\
2 \\
+1 \\
$\infty$ \\
$\infty$ \\
-1 \\
1 \\
\end{tabular} & $\begin{array}{l}\overrightarrow{0} \\
\stackrel{0}{0} \\
\dot{0} \\
0 \\
0 \\
0 \\
0\end{array}$ & 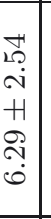 & 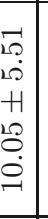 & & 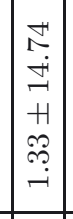 & 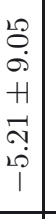 & $\begin{array}{l}\hat{1} \\
1 \\
10 \\
+1 \\
0 \\
0 \\
0 \\
\end{array}$ & 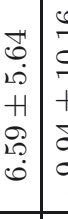 \\
\hline & 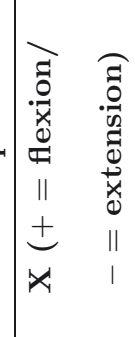 & 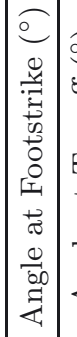 & 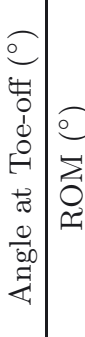 & 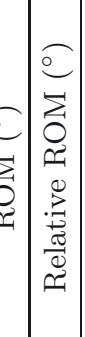 & 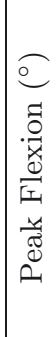 & 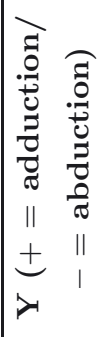 & 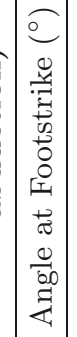 & 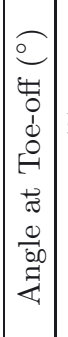 & $\left|\begin{array}{l}0 \\
0 \\
\vdots \\
0 \\
x^{2}\end{array}\right|$ & 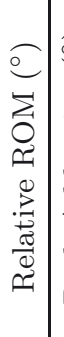 & 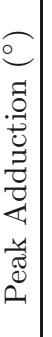 & 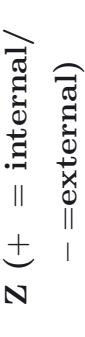 & 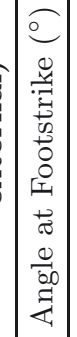 & 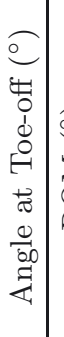 & 范 & 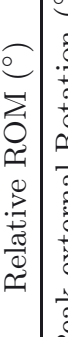 \\
\hline
\end{tabular}

$\frac{0}{0} \stackrel{0}{\oplus}$ 


\begin{tabular}{|c|c|c|c|c|c|c|c|c|c|c|c|c|c|c|c|c|c|c|}
\hline$\gamma$ & & 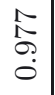 & $\begin{array}{l}20 \\
2 \\
8 \\
O \\
0\end{array}$ & $\begin{array}{l}\mathscr{D} \\
\stackrel{0}{O} \\
0\end{array}$ & $\begin{array}{l}\mathscr{8} \\
\stackrel{\circ}{\circ}\end{array}$ & $\begin{array}{l}10 \\
5 \\
0 \\
0\end{array}$ & & $\mid \begin{array}{l}0 \\
0 \\
0 \\
0 \\
0\end{array}$ & $\mid \begin{array}{l}\overrightarrow{0} \\
0 \\
0 \\
0\end{array}$ & $\begin{array}{l}\infty \\
\approx \\
O \\
0\end{array}$ & \begin{tabular}{l}
0 \\
\multirow{2}{*}{} \\
0 \\
0
\end{tabular} & \begin{tabular}{|l|l}
0 \\
0 \\
0 \\
0 \\
0
\end{tabular} & & \begin{tabular}{|l}
2 \\
2 \\
0 \\
0 \\
0
\end{tabular} & $\begin{array}{l}0 \\
0 \\
\infty \\
0 \\
0\end{array}$ & \begin{tabular}{|l}
$\mathscr{2}$ \\
$\mathscr{2}$ \\
0 \\
0 \\
0
\end{tabular} & 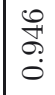 & $\mid \begin{array}{l}\infty \\
\infty \\
\infty \\
0 \\
0\end{array}$ \\
\hline 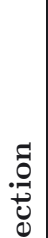 & $\begin{array}{l}\vec{v} \\
0 \\
\overrightarrow{0} \\
\overrightarrow{0} \\
\vec{n}\end{array}$ & 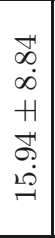 & $\begin{array}{c}18 \\
0 \\
1 \\
+1 \\
10 \\
10 \\
0 \\
-9 \\
-1\end{array}$ & 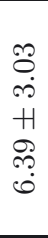 & $\begin{array}{l}0 \\
0 \\
0 \\
+1 \\
0 \\
0 \\
1 \\
0\end{array}$ & $\begin{array}{l}\text { N } \\
\dot{\sigma} \\
+ \\
+ \\
0 \\
0 \\
0 \\
\dot{H}\end{array}$ & & $\begin{array}{l}0 \\
+ \\
+ \\
i \\
+ \\
H \\
+ \\
+ \\
0 \\
0\end{array}$ & $\mid \begin{array}{l}0 \\
0 \\
0 \\
i \\
+1 \\
0 \\
0 \\
0 \\
0 \\
1\end{array}$ & $\begin{array}{c}\vec{b} \\
i \\
+ \\
+ \\
\dot{b} \\
\dot{v}\end{array}$ & 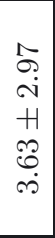 & 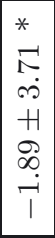 & & $\begin{array}{l}\not \\
\infty \\
20 \\
10 \\
+1 \\
0 \\
10 \\
0 \\
1 \\
1\end{array}$ & $\begin{array}{l}\infty \\
0 \\
\infty \\
+1 \\
+1 \\
0 \\
\infty \\
0 \\
1\end{array}$ & $\begin{array}{l}\mathcal{N} \\
\stackrel{\sim}{\sim} \\
+H \\
\mathcal{N} \\
\stackrel{N}{+}\end{array}$ & 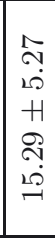 & 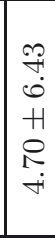 \\
\hline $\begin{array}{l}0 \\
\dot{0} \\
0\end{array}$ & 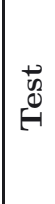 & 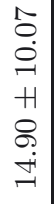 & $\begin{array}{l}0 \\
\infty \\
\sim \\
+1 \\
+ \\
R \\
\infty \\
\infty \\
-1\end{array}$ & $\begin{array}{l}\vec{\sim} \\
\ddot{\infty} \\
\dot{H} \\
\stackrel{m}{\leftrightarrow} \\
\stackrel{\leftrightarrow}{0}\end{array}$ & 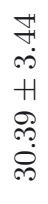 & 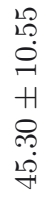 & & $\begin{array}{l}12 \\
1 \\
i \\
i 1 \\
1 \\
20 \\
0 \\
i \\
1\end{array}$ & $\mid \begin{array}{c}\vec{H} \\
10 \\
i \\
+1 \\
0 \\
0 \\
0 \\
0 \\
i\end{array}$ & 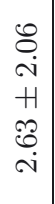 & $\begin{array}{l}8 \\
\dot{\infty} \\
\dot{H} \\
\dot{\infty} \\
\dot{\infty}\end{array}$ & $\begin{array}{c}1 \\
0 \\
\infty \\
0 \\
+1 \\
0 \\
0 \\
10 \\
1 \\
1\end{array}$ & & 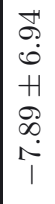 & $\begin{array}{l}\infty \\
0 \\
\infty \\
+1 \\
0 \\
0 \\
10 \\
10 \\
1\end{array}$ & 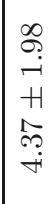 & 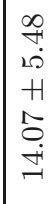 & 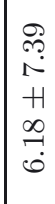 \\
\hline$\gamma$ & & $\begin{array}{l}12 \\
5 \\
0 \\
0\end{array}$ & $\begin{array}{l}\vec{F} \\
\stackrel{\sigma}{\sigma} \\
O\end{array}$ & $\begin{array}{l}\mathscr{8} \\
\stackrel{\circ}{\circ}\end{array}$ & $\begin{array}{l}\mathscr{D} \\
\infty \\
O \\
0\end{array}$ & $\begin{array}{l}\infty \\
\infty \\
\infty \\
0 \\
0\end{array}$ & & $\mid \begin{array}{l}-\overrightarrow{0} \\
0 \\
0\end{array}$ & $\mid \begin{array}{l}\mathbb{N} \\
\stackrel{N}{0} \\
0\end{array}$ & $\begin{array}{l}0 \\
\infty \\
\stackrel{0}{0}\end{array}$ & $\begin{array}{l}\vec{H} \\
\text { హ! } \\
0\end{array}$ & $\mid \begin{array}{l}0 \\
0 \\
0 \\
0\end{array}$ & & \begin{tabular}{|l}
12 \\
$\stackrel{1}{+}$ \\
0 \\
0
\end{tabular} & $\mid \begin{array}{l}0 \\
0 \\
\infty \\
0\end{array}$ & \begin{tabular}{|l}
$\vec{\infty}$ \\
0 \\
0 \\
0
\end{tabular} & F̆ & $\begin{array}{l}2 \\
\infty \\
\infty \\
0 \\
0\end{array}$ \\
\hline $\begin{array}{l}\varpi ే \\
. \\
.\end{array}$ & 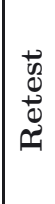 & $\begin{array}{l}\tilde{0} \\
\dot{0} \\
+ \\
\dot{\sigma} \\
\dot{\sigma}\end{array}$ & $\begin{array}{l}\infty \\
0 \\
10 \\
+1 \\
-1 \\
\overrightarrow{10} \\
\dot{9} \\
-1\end{array}$ & $\begin{array}{l}\overrightarrow{0} \\
\dot{\infty} \\
+1 \\
\stackrel{0}{0} \\
\dot{0}\end{array}$ & $\begin{array}{l}\stackrel{\sim}{N} \\
\text { N丶 } \\
+1 \\
0 \\
\stackrel{1}{0} \\
0\end{array}$ & 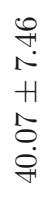 & & 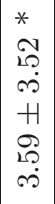 & 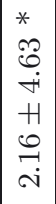 & 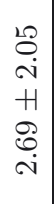 & $\begin{array}{l}2 \\
\infty \\
o \dot{0} \\
+ \\
+ \\
\dot{H} \\
\dot{+}\end{array}$ & $\begin{array}{l}* \\
* \\
\llcorner \\
0 \\
+ \\
+ \\
+1 \\
\vec{H} \\
\dot{0}\end{array}$ & & $\begin{array}{l}0 \\
1 \\
20 \\
1 \\
0 \\
0 \\
1 \\
0 \\
1\end{array}$ & 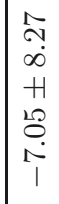 & 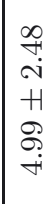 & 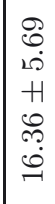 & $\begin{array}{l}20 \\
10 \\
1 \\
+1 \\
0 \\
0 \\
0 \\
0 \\
0\end{array}$ \\
\hline$\underset{\substack{0 \\
\Xi}}{0}$ & 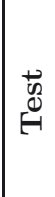 & $\begin{array}{l}\infty \\
0 \\
i \\
+ \\
+1 \\
\stackrel{0}{0} \\
\sigma\end{array}$ & 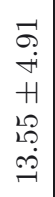 & $\begin{array}{l}\vec{N} \\
\hat{n} \\
+H \\
\hat{\infty} \\
\hat{n} \\
0\end{array}$ & 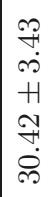 & 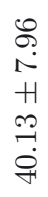 & & 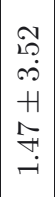 & $\begin{array}{c}\mathscr{g} \\
\dot{+} \\
+ \\
+1 \\
\infty \\
+1 \\
\dot{1}\end{array}$ & 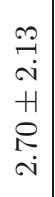 & 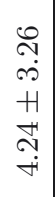 & 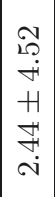 & & $\begin{array}{l}\infty \\
0 \\
1 \\
+1 \\
+1 \\
\tilde{\alpha} \\
0 \\
1 \\
1\end{array}$ & 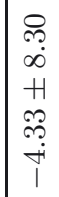 & 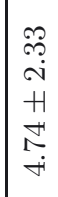 & 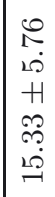 & 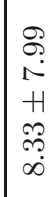 \\
\hline$\sigma$ & & $\begin{array}{l}\infty \\
0 \\
5 \\
0\end{array}$ & 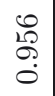 & $\mid \begin{array}{l}0 \\
\infty \\
0 \\
0 \\
0\end{array}$ & $\vec{\sigma}$ & $\mid \begin{array}{l}\overrightarrow{0} \\
0 \\
0 \\
0\end{array}$ & & $\mid \begin{array}{l}0 \\
\mathscr{D} \\
0 \\
0 \\
0\end{array}$ & $\begin{array}{l}0 \\
\stackrel{N}{1} \\
0 \\
0\end{array}$ & $\begin{array}{l}+1 \\
\infty \\
\stackrel{0}{0} \\
0\end{array}$ & $\begin{array}{l}\infty \\
2 \\
8 \\
0 \\
0\end{array}$ & $\begin{array}{l}0 \\
0 \\
0 \\
0 \\
0\end{array}$ & & $\begin{array}{l}\hat{2} \\
\hat{2} \\
0\end{array}$ & 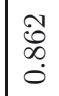 & \begin{tabular}{|l}
10 \\
0 \\
0 \\
0 \\
0
\end{tabular} & 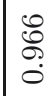 & $\begin{array}{l}\hat{N} \\
\tilde{\infty} \\
0\end{array}$ \\
\hline 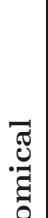 & 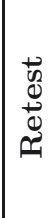 & $\begin{array}{l}\vec{H} \\
\overrightarrow{0} \\
+ \\
\tilde{O} \\
\tilde{O} \\
\dot{-}\end{array}$ & 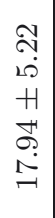 & $\begin{array}{l}\tilde{0} \\
\dot{\rho} \\
\dot{H} \\
\hat{\sigma} \\
0 \\
0\end{array}$ & 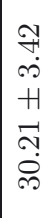 & 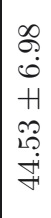 & & $\begin{array}{l}0 \\
0 \\
o \\
H \\
H \\
\exists \\
-1 \\
-1\end{array}$ & 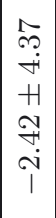 & 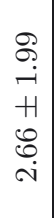 & $\begin{array}{l}\mathscr{D} \\
\infty \\
i \\
+H \\
\dot{H} \\
\dot{\theta} \\
\dot{\infty}\end{array}$ & $\begin{array}{c}\infty \\
\infty \\
+ \\
+ \\
+1 \\
0 \\
0 \\
\dot{1} \\
1 \\
1\end{array}$ & & 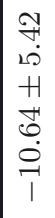 & 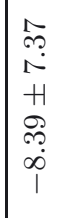 & 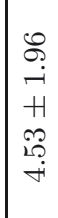 & 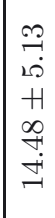 & 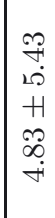 \\
\hline 疍 & 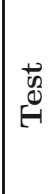 & 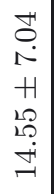 & 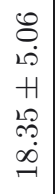 & 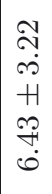 & 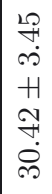 & 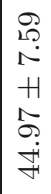 & & 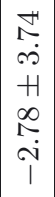 & $\begin{array}{c}\vec{\infty} \\
0 \\
\dot{0} \\
+1 \\
0 \\
0 \\
0 \\
0 \\
1\end{array}$ & 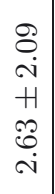 & 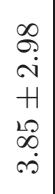 & \begin{tabular}{l}
0 \\
$\sim$ \\
+ \\
+ \\
+ \\
+ \\
\hdashline \\
0 \\
0 \\
1
\end{tabular} & & 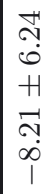 & $\begin{array}{l}\vec{H} \\
\stackrel{+}{+} \\
+ \\
\stackrel{0}{0} \\
\dot{0} \\
1\end{array}$ & 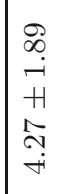 & 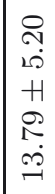 & $\begin{array}{l}\mathscr{2} \\
\dddot{0} \\
0 \\
H \\
\ddot{0} \\
\ddot{0}\end{array}$ \\
\hline تئدِ & 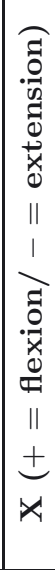 & $\begin{array}{l}\sigma \\
0 \\
0 \\
.4 \\
0 \\
0 \\
0 \\
0 \\
0 \\
0 \\
0 \\
0 \\
00 \\
0\end{array}$ & 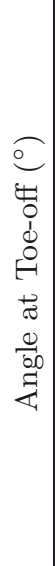 & 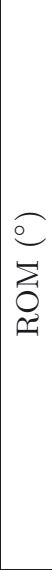 & 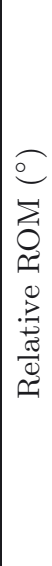 & 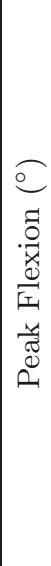 & 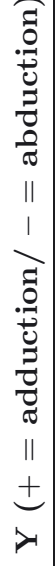 & 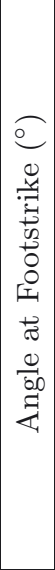 & 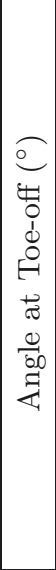 & $\begin{array}{l}\text { } \\
\sum_{0} \\
0 \\
0\end{array}$ & 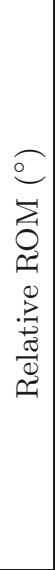 & 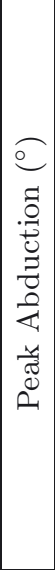 & 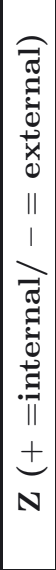 & 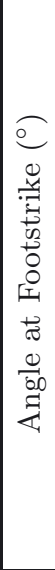 & 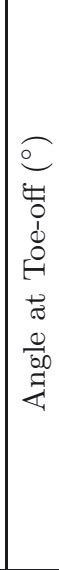 & 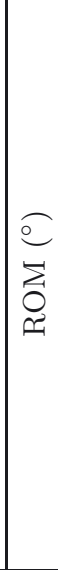 & 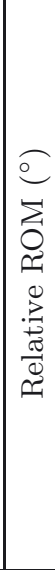 & 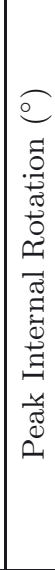 \\
\hline
\end{tabular}



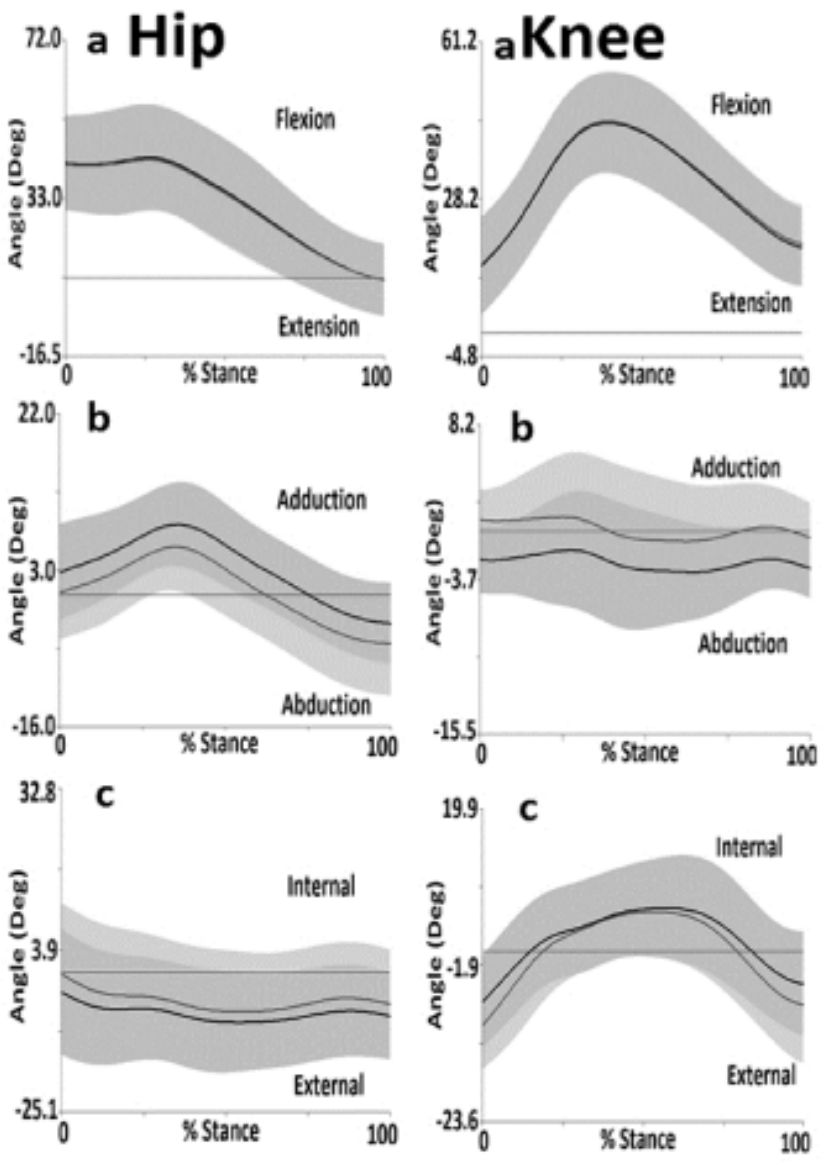

Fig. 4. Hip and knee joint kinematics from the projection HJC in the a. sagittal, b. coronal and c. transverse plane as a function of test (black) and retest (grey) static trials.

\section{Discussion}

The aim of the current investigation was to determine the reliability of the three principal methods of defining the HJC. In the present study, running trials were analysed simultaneously using three different anatomical coordinate systems. This represents the first study investigating the test-retest reliability in defining the HJC using the anatomical, projection and functional techniques via discrete kinematic parameters and waveform analyses.

In the sagittal plane no differences were observed between test and retest angular parameters for any of the three HJC locations techniques. In addition to this the highest intra-class correlations were observed for the sagittal plane waveforms indicating a high level of reliability for all techniques in the sagittal plane. This observation concurs with the observations of Besier, et al. (2003) who noted that kinematics in the sagittal plane are known to be less sensitive to alterations in joint centre locations in comparison to the coronal and transverse planes.

However in the coronal and transverse planes significant differences were observed between test and retest angular parameters using both the functional and projection techniques. Notably, however the anatomical technique was associated with no significant test-retest differences in either the coronal and transverse planes. This finding has potential clinical significance for researchers using the projection of functional techniques as increases in transverse and coronal plane rotations of the hip in conjunction with increases in knee coronal plane movements may facilitate increases in dynamic Q-angle during running (Horton \& Hall, 1989; Hreljac, Marshall, \& Hume, 2000; Hsu, et al., 1990) Increases in dynamic Q-angle are implicated in the aetiology of patellofemoral pathologies (Mizuno, et al., 2001) Therefore, when performing longitudinal analyses in subject groups such as the current investigation it may be prudent to select the anatomical hip joint centre location technique in order for empirically meaningful conclusions to be made.

The reliability analyses of both discrete variables using cronbach's alpha and waveforms using intra-class correlations correspond with the statistical examination using paired t-tests in that the anatomical technique was associated with the highest intra-class correlations between test-retest waveforms in comparison to the functional and projection techniques. This finding counters the hypotheses of Besier, et al. (2003) who postulate that techniques for joint centre location using anatomical markers have greater propensity for error from marker re-application. There are several potential mechanisms for this observation; firstly the examiner performing the analyses had extensive experience in locating anatomical positions around the pelvis, thus facilitating the increased reliability in defining the HJC using the anatomical technique. Furthermore, all participants in this examination were trained distance runners with minimal body fat, which facilitated the examiners ability to reliability locate anatomical landmarks. Finally, it is hypothesized that this finding may relate to the difficulty in re-producing the same range of movements required to locate the HJC using the functional method thus potentially compromising its reliability in comparison to the anatomical technique. It should be noted however that the precision and accuracy with which the HJC location is determined using the functional method has been shown to be sensitive to a number of analytical and experimental factors. Thus that the current investigation utilized a single technique for the functional HJC definition may serve as a slight limitation to the current investigation. It is recommended that future work consider the test-retest reliability of the available functional techniques to identify the HJC.

That the projection technique was associated with the lowest levels of reliability is perhaps unsurprising despite the observations of Weinhandl and O'Connor (2010). The head of the greater trochanter is broad in comparison to other anatomical locations and difficulty in defining this landmarks as a mere point, was documented by Della Croce, et al. (1999) who reported test-retest errors of $12.2 \mathrm{~mm}$ in the anteroposterior direction in locating the greater trochanter marker. This potential mislocation 
between test-retest static trials would influence the results kinematic waveforms, particularly in the coronal and transverse planes.

The enhanced repeatability of the functional model compared to the projection technique is likely to become more pronounced with examiners who are less experienced or in overweight participant groups and with subjects who have skeletal pathologies whereby the location of anatomical landmarks becomes more difficult. Furthermore, in situations where location of skeletal landmarks is difficult it may be preferable to select the functional technique in favour of the anatomical technique which would also be influenced by these factors. These issues clearly require further investigation and it may be prudent to repeat the current investigation using less experienced examiners and a pathological participant group.

Neither the anatomical or projection techniques appear to have the same propensity for error from marker re-application as the examinations conducted by Kadaba, et al. (1989) and Growney, et al. (1997). This probably relates to their utilization use of mid-segment markers to define joint centres and axes of rotation models may explain the low repeatability in non-sagittal plane data compared to the projection and anatomical model presented here. Finally although differences in reliability were evident between the three techniques, it is unclear from the observations of the current study which method had greater reliability in closely estimating the actual HJC location. Future work should therefore be conducted which validate these techniques using radiographical techniques.

Nonetheless the results of this investigation suggest that the anatomical technique for the estimation of the HJC appears to be the most appropriate technique for experienced practitioners examining a non-pathological sample, and as such its utilization for running analyses is recommended.

\section{Bibliography}

Andriacchi, T.P., Anderson, G.B., Fermier, R.W., Stern, D., \& Galante, J.O. (1980). A study of lower-limb mechanics during stair-climbing. Journal of Bone Joint Surgery, 62, 749-757.

Bell, A.L., Brand, R.A., \& Pedersen, D.R. (1999). Prediction of hip joint centre location from external landmarks. Human Movement Science, 8, 3-16.

Besier, T.F., Sturnieks, D.L, Alderson, J.A., \& Lloyd, D.G. (2003). Repeatability of gait data using a functional hip joint centre and a mean helical knee axis. Journal of Biomechanics, 36, 1159-1168.

Cappozzo, A. (1984). Gait analysis methodology. Human Movement Science, 3, 27-50.

Capozzo, A., Leo T. \& Pedotti, A. (1975). A general computing method for the analysis of human locomotion. Journal of Biomechanics, 8, 307-320.
Cappozzo, A., Catani, F., Leardini, A., Benedeti, M.G., \& Della, C.U. (1995). Position and orientation in space of bones during movement: Anatomical frame definition and determination. Clinical Biomechanics, 10, 171-178.

Cappozzo, A., Cappello, A., Croce U., \& Pensalfini F. (1997). Surface-marker cluster design criteria for 3-D bone movement reconstruction. IEEE Transactions on Biomedical Engineering, 44, 1165-1174.

Della Croce, U., Cappozzo, A., \& Kerrigan, D.C. (1999). Pelvis and lower limb anatomical landmark calibration precision and its propagation to bone geometry and joint angles. Medical Biological Engineering Computing, 37, $155-161$.

Growney, E., Meglan, D., Johnson, M., Cahalan, T., \& An, K. (1997). Repeated measures of adult normal walking using a video tracking system. Gait \& Posture, 6, 147-162.

Horton, M.G., \& Hall, T.L. (1989). Quadriceps femoris muscle angle: normal values and relationships with gender and selected skeletal measures. Physical Therapy, 69, 897-901.

Hreljac, A., Marshall, R.N. \& Hume, P.A. (2000). Evaluation of lower extremity overuse injury potential in runners. Medecine and Science in Sport and Exercise, 32, 1635-1641.

Hsu, R.W., Himeno, S., Coventry, M.B., \& Chao, E.Y. (1990). Normal axial alignment of the lower extremity and loadbearing distribution at the knee. Clinical Orthopaedics and Related Research, 255, 215-227.

Kadaba, M.P., Ramakrishnan, H.K., Wootten, M.E., Gainey, J., Gorton, G., \& Cochran, G.V. (1989). Repeatability of kinematic, kinetic, and electromyographic data in normal adult gait. Journal of Orthopaedic Research, 7, 849-860.

Kirkwood, R.N., Culham, E.G., \& Costigan, P. (1999). Radiographic and non-invasive determination of the hip joint center location: effect on hip joint moments. Clinical Biomechanics, 14, 227-235.

Leardini, A., Cappozzo, A., Catani, F., Toksvig-Larsen, S., Petitto, A., Sforza, V., Cassanelli, G., \& Giannini, S. (1999). Validation of a functional method for the estimation of hip joint centre location. Journal of Biomechanics, 32, 99-103.

Marin, F., Mannel, H., Claes, L., \& Durselen, L. (2003). Accurate determination of a joint rotation center based on the minimal amplitude point method. Computer Aided Surgery, 8, 30-34.

Mizuno, Y., Kumagai, M., Mattessich, S.M., Elias, J.J., Ramrattan, N., Cosgarea, A.J., \& Chao, E.Y. (2001). Qangle influences tibiofemoral and patellofemoral kinematics. Journal of Orthopaedic Research, 19, 834-840.

Piazza, S.J., Okita, N., \& Cavanagh, P.R. (2001). Accuracy of the functional method of hip joint center location: effects of limited motion and varied implementation. Journal of Biomechanics, 34, 967-973.

Rothman, K.J. (1990). No adjustments are needed for multiple comparisons. Epidemiology, 1, 43-46. 
Schwartz, M.H., \& Rozumalski, A. (2005). A new method for estimating joint parameters from motion data. Journal of Biomechanics, 38, 107-116.

Seidel, G.K., Marchinda, D.M., Dijkers, M., \& Soutas-Little, R.W. (1995). Hip joint center location from palpable bony landmarks - a cadaver study. Journal of Biomechanics, 28, 995-998.

Shea, K.M., Lenhoff, M.W., Otis, J.C., \& Backus, S.I. (1997). Validation of a method for location of the hip joint center. Gait 6 Posture, 5, 157-158.

Sinclair, J., Edmundson, C.J., Brooks, D., \& Hobbs, S.J. (2011). Evaluation of kinematic methods of identifying gait Events during running. International Journal of Sport Science and Engineering, 5, 188-192.

Stagni, R., Leardini, A., Cappozzo, A., Grazia Benedetti, M., \& Cappello, A. (2000). Effects of hip joint centre mislocation on gait analysis results. Journal of Biomechanics, 33, 1479-1487.

Tylkowski, C.M., Simon, S.R., \& Mansour, J.M. (1982). Internal rotation gait in spastic cerebral palsy. The Hip, 89-125.

Weinhandl, J.T. \& O'Connor, K.M. (2010). Assessment of a greater trochanter-based method of locating the hip joint center. Journal of Biomechanics, 43, 2633-2636. 